Images dans le monde ibérique et ibéricoaméricain

$6 \mid 2013$

Images dans le monde ibérique et ibéro-américain

\title{
Santo à La Havane : vagabondages cinématographiques d'un héros mexicain
}

\section{Emmanuel Vincenot}

\section{(2) OpenEdition}

10 Journals

Édition électronique

URL : http://journals.openedition.org/agedor/1102

DOI : 10.4000 /agedor.1102

ISSN : 2104-3353

Éditeur

Laboratoire LISAA

Référence électronique

Emmanuel Vincenot, «Santo à La Havane : vagabondages cinématographiques d'un héros mexicain », L'Âge d'or [En ligne], 6 | 2013, mis en ligne le 01 mars 2013, consulté le 15 septembre 2020. URL : http://journals.openedition.org/agedor/1102 


\section{Santo à La Havane : vagabondages cinématographiques d'un héros mexicain}

Résumé : L’une des formules génériques les plus durables du cinéma mexicain éclot au milieu des années 1950, alors que l'âge d'or des studios touche à sa fin ; elle met en scène les héros de la lucha libre, ce sport-spectacle qui rassemble alors des millions de fidèles chaque semaine. Après avoir contextualisé l'émergence du cine de luchadores, l'article retrace la trajectoire cinématographique de Santo, la plus grande vedette du genre, et se concentre sur les deux premiers opus de sa filmographie: Santo contra cerebro del mal [Joselito Rodríguez, 1961] et Santo contra hombres infernales [Joselito Rodríguez, 1961]. Ces deux longs métrages tournés à La Havane sont particulièrement représentatifs du cinéma dit de mexploitation et se caractérisent par une désinvolture formelle qui atteint une dimension quasiment expérimentale, mettant à nu les ressorts mêmes du langage cinématographique.

Mots-clés : Cinéma - Mexique - Santo - Catch - Mexploitation - Recyclage - Cuba

Resumen: Una de las fórmulas genéricas más exitosas del cine mexicano apareció a mediados de los años 1950, cuando la edad de oro de los estudios se estaba terminando. Ponía en escena a los héroes de la lucha libre, aquel deporte-espectáculo que reunía entonces a millones de seguidores cada semana. Después de contextualizar la emergencia del cine de luchadores, el artículo evoca la trayectoria cinematográfica de Santo, la mayor estrella del género y se concentra en los dos primeros títulos de su filmografía : Santo contra cerebro del mal [Joselito Rodríguez, 1961] y Santo contra hombres infernales [Joselito Rodríguez, 1961]. Ambos largometrajes, filmados en La Habana, son muy representativos del llamado "mexplotación cinema» y se caracterizan por una falta de cuidado formal que alcanza una dimensión casi experimental, desvelando los entresijos mismos del lenguaje cinematográfico.

Palabras clave: Cine - México - Santo - Lucha libre - Mexploitation - Reciclaje - Cuba

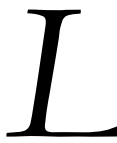

os Olvidados est assurément l'un des films latino-américains les plus célèbres des années 1950, mais la postérité du chef-d'œuvre de Luis Buñuel ne doit pas faire oublier le riche panorama du cinéma mexicain de l'époque, où les grosses productions léchées côtoyaient les navets tournés à la va-vite. Bâti sur le modèle hollywoodien, ce cinéma cherche alors à s'attirer les faveurs d'un public de plus en plus urbain et avide de loisirs bon marché, en lui proposant une palette variée de genres filmiques dont certains sont importés des États-Unis (comme le film de gangsters) mais dont la plupart ont une origine locale. C'est le cas par exemple de la comedia ranchera, née en 1936 avec Alla en el rancho grande [Fernando de Fuentes]: le film rencontra un succès tellement phénoménal qu'il permit à lui seul l'éclosion d'un cinéma industriel au cours des années suivantes; mais il faut également mentionner le cine de rumberas, qui exploitait la plastique de jeunes danseuses de cabaret, ou la comedia arrabalera, vouée à l'évocation très 
édulcorée de la vie des habitants des quartiers populaires de Mexico (un genre d'ailleurs détourné et critiqué par Buñuel dans Los Olvidados). Chacun connut son heure de gloire avant de décliner, l'industrie cherchant systématiquement à remplacer une formule moribonde par une nouvelle proposition générique susceptible de ressusciter l'intérêt toujours vacillant du public (n'oublions pas que le cinéma repose sur une économie de l'offre, et non de la demande ${ }^{1}$ ). Une grande partie de l'histoire du cinéma mexicain peut ainsi s'écrire en fonction des différents genres qui se sont succédé ou chevauchés depuis l'apparition de la comedia ranchera dans les années 1930.

L'une des formules génériques les plus durables et les plus populaires éclot au milieu des années 1950, alors que l'âge d'or des studios mexicains touche à sa fin; elle met en scène les héros du catch local, la lucha libre, ce sport-spectacle qui rassemble alors des millions de fidèles chaque semaine. Pendant une trentaine d'années, près de 200 films de luchadores seront produits et diffusés, non seulement au Mexique mais également à l'étranger ; parmi tous ces titres, plus d'une cinquantaine mettront en scène Santo, alias «El enmascarado de plata ", lutteur au succès inégalé, qui figure aujourd'hui au panthéon de la culture populaire mexicaine et latino-américaine. Après avoir contextualisé l'émergence de ce cine de luchadores, je m’intéresserai plus précisément à la trajectoire cinématographique de Santo, en me concentrant sur les deux premiers opus de son impressionnante filmographie: Santo contra cerebro del mal [Joselito Rodríguez, 1961] et Santo contra hombres infernales [Joselito Rodríguez, 1961], deux longs métrages tournés à La Havane, qui présentent un certain nombre de caractéristiques originales par rapport aux autres films de la série.

\section{Naissance d'un genre}

Les films de catch mexicains apparaissent au milieu des années 1950, alors que la lucha libre jouit d'une très grande popularité. Les producteurs de cinéma cherchent alors à exploiter de manière parasitaire cet engouement du grand public, comme ils l'ont déjà fait avec les danseuses de cabarets, le folklore tapatío ou la radionovela, et ils conçoivent leurs films comme des produits dérivés d'une forme culturelle préexistante. En retour, ces productions contribuent à renforcer ce dont elles s'inspirent.

Les débuts de la lucha libre remontent à la fin du XIX ${ }^{\mathrm{e}}$ siècle, mais c'est surtout à partir des années 1910-1920 que ce sport théâtral s'enracine dans les goûts et les pratiques culturelles des classes populaires mexicaines. Au début, les spectacles s'inspirent en grande partie du catch tel qu'on le pratique en Europe et aux États-Unis, et la plupart des lutteurs sont d'ailleurs étrangers, très souvent américains. Progressivement, néanmoins, la lucha libre se nationalise et se singularise. Au début des années 1930, les catcheurs mexicains finissent par évincer les lutteurs étrangers dans le cœur du public local, aidés en cela par les autorités qui limitent la présence des non-nationaux sur les rings. Tout en conservant le mélange de défi sportif et de théâtre de Grand-Guignol qui fait l'originalité de la discipline, les lutteurs

\footnotetext{
${ }^{1}$ Sur les aspects économiques du cinéma, lire : CRETON, Laurent, Économie du cinéma : perspectives stratégiques, Paris, Ed. Armand Colin, 2009.
} 
mexicains amènent une nouveauté fondamentale : le masque ${ }^{2}$. Cet ajout, qui semble avoir d'abord été introduit épisodiquement, en 1934, par un lutteur appelé Luis Núñez, avant que El Murciélago Enmascarado ne l'impose systématiquement en $1938^{3}$, s'inscrit pleinement dans la logique théâtrale du catch, qui veut que chaque lutteur incarne un personnage immédiatement reconnaissable, et permet également de proposer des modèles d'identifications fédérateurs à des publics très hétérogènes (provinciaux, chilangos ${ }^{4}$, Blancs, indigènes, etc. $\left.{ }^{5}\right)$ tout en puisant aux sources des cultures locales précolombiennes (le masque du luchador renvoie aussi bien aux masques funéraires mayas et teotihuacanos qu'aux imposantes têtes olmèques). Comme le fait d'ailleurs remarquer Evan Lieberman, «the mask might well be considered the defining icon of Mexican culture $»^{6}$.

D’abord cantonnée aux arrière-cours et aux ruelles malfamées, la lucha libre gagne progressivement en visibilité et respectabilité, sans cesser pour autant d'être un divertissement prisé essentiellement par les couches populaires. Dans les années 1930, les premiers espaces exclusivement dédiés à ce spectacle, désignés sous le terme de arenas, se multiplient à Mexico, chaque colonia prolétaire comptant son arène. Comme le souligne Álvaro Fernández,

la lucha libre se convierte entonces en espectáculo de barrio, es reguladora de tensiones, bálsamo de la pobreza. La gente de los espacios populares tiene a su alcance un sitio donde puede cumplirse la promesa de justicia en la reiteración del rito: la representación eterna de la lucha del bien contra el mal. El escenario de las luchas es el recinto sagrado para la ceremonia, es una pirámide invertida que nos remite de alguna manera a determinados rituales prehispánicos ${ }^{7}$.

Grâce à l'impulsion décisive d'un entrepreneur, Salvador Lutteroth, l'ouverture régulière de salles pouvant contenir plusieurs centaines, voire plusieurs milliers de spectateurs (arenas Modelo, México, Nacional, Coliseo, etc.), à Mexico puis dans les grandes villes de province, permet de drainer des foules de plus en plus importantes et de développer l'économie de la lucha libre, qui devient une activité de première importance dans la société des loisirs naissante. L'essor financier de la discipline ainsi que sa visibilité croissante attirent des candidats de plus en plus nombreux à la carrière de lutteur, parmi lesquels Rodolfo Guzmán Huerta, le futur Santo. Des centaines de luchadores rivalisent alors d'audace et d'imagination pour attirer l'attention du public, s'inventant des noms d'artistes rocambolesques et arborant des costumes parfois spectaculaires. Leurs personnages puisent à toutes les sources de l'imaginaire populaire (qu'elles soient religieuses, mythologiques, folkloriques, littéraires, cinématographiques) et forment un panthéon foisonnant, mais strictement réparti entre bons (les técnicos, également appelés cientificos) et méchants (les rudos). Les premiers respectent les règles et font preuve d'esprit chevaleresque tandis que les seconds sont prêts à toutes les bassesses et toutes les traîtrises pour remporter la victoire.

\footnotetext{
${ }^{2}$ L'utilisation des masques amène parfois à comparer le panthéon de la lucha libre mexicaine à celui des superhéros de la bande dessinée américaine, mais c'est plutôt du côté de Zorro, héros très humain, dépourvu de super-pouvoirs et créé en 1919 par Johnston McCulley, qu'il faut chercher une filiation, si filiation il y a.

3 Cf. FERNÁNDEZ, Álvaro A., Santo, el Enmascarado de Plata. Mito y realidad de un béroe mexicano moderno, Guadalajara (Mexique), Editorial Universitaria, 2012, p. 87-88.

4 « Habitants de la capitale », en espagnol familier du Mexique.

${ }^{5}$ Cf. ibid., p. 48.

${ }^{6}$ LIEBERMAN, Evan, "Mask and Masculinity: Culture, Modernity, and Gender Identity in the Mexican Lucha Libre films of El Santo » in Studies in Hispanic Cinemas, Vol. 6, n¹, Londres, Ed. Intellect, 2009, p. 3.

${ }^{7}$ FERNÁNDEZ, Álvaro A., op. cit. , p. 48.
} 
Lors des matchs de lucha libre, cette division binaire des rôles, jouée avec emphase, excite les passions de la foule, qui s'invite dans le spectacle en apostrophant les lutteurs, pour les insulter ou les encourager ${ }^{8}$. À la fin du spectacle, les lutteurs rejoignent les vestiaires, quittent leurs masques et retournent à l'anonymat de la vie civile après avoir enflammé l'arène pendant des heures.

L'influence sociale grandissante de la lucha libre dans les années 1950 amena les autorités à s'inquiéter du phénomène, selon la logique qui veut que certaines formes culturelles populaires nouvelles, en particulier celles qui font intervenir la représentation de la violence, suscitent une réaction de peur au sein des classes dominantes (les Anglo-Saxons désignent le phénomène par le terme de moral panic'). En 1953, alors que la télévision faisait ses premiers pas, l'État interdit aux chaînes mexicaines de retransmettre des matchs de catch afin de protéger la jeunesse de leur influence jugée pernicieuse (la décision prit effet alors que plusieurs retransmissions avaient déjà eu lieu, rencontrant un grand succès). La mesure n'empêcha pas la lucha libre de prospérer et d'augmenter son audience par d'autre biais, en particulier grâce au cinéma. Tandis que ce divertissement populaire connaissait un véritable âge d'or, le cinéma mexicain, pour sa part, voyait le sien prendre fin et quelques producteurs précurseurs, à la recherche de nouveaux filons à exploiter après qu'ils eussent épuisé celui des comedias rancheras et des films de rumberas, commencèrent à s'intéresser au potentiel commercial du catch. L'univers de la lucha libre offrait en effet un large éventail de vedettes prêtes à l'emploi, un monde de fantaisie et d'aventures, à la fois très codifié et hautement iconique : l'association avec le $7^{\text {ème }}$ art semblait naturelle.

Néanmoins, le genre du cine de luchadores ne prit pas forme d'un seul coup, comme cela avait été le cas avec la comedia ranchera, mais se dessina progressivement, par apports successifs. Les premières manifestations cinématographiques débutent en 1952, année où sont tournés quatre films dont la sortie s'étale jusqu'en 1954 (il faut d'ailleurs souligner la concordance presque parfaite entre l'interdiction de la retransmission télévisée des matchs de catch et la naissance du film de luchadores, comme si l'industrie cinématographique mexicaine avait saisi au bond la possibilité de fournir au public des images que l'on souhaitait lui empêcher de voir par ailleurs). Le premier film à être distribué est curieusement une comédie, qui aborde l'univers du catch sur le mode burlesque. Il s'agit de El luchador fenómeno [Fernando Cortés, 1952], sorti le 17 octobre 1952, avec l'acteur Resortes en vedette, qui reprend là la recette de son film précédent El beisbolista fenómeno [Fernando Cortés, 1952]. Les titres suivants évoquent la lucha libre sur un mode plus sérieux, mais restent dépendants d'un genre préexistant. Dans La bestia magnifica [Chano Urueta, 1953], qui raconte l'histoire de deux amis qui rêvent de devenir catcheurs, le traitement est mélodramatique, une caractéristique que l'on retrouve dans Huracán Ramírez. [Joselito Rodríguez, 1953], où le personnage central, un luchador imaginaire (les producteurs n'ont pas fait appel à un véritable lutteur fréquentant les rings) cherche avant tout à aider son

\footnotetext{
${ }^{8}$ Il faut également mentionner le personnage de l'arbitre : généralement incompétent ou corrompu, il ne parvient que rarement à faire respecter les règles par le rudo, ce qui complique la tâche du técnico. Le spectacle de la lucha libre renvoie donc très clairement à la vie quotidienne de la plupart des Mexicains, confrontés à des autorités (politiques, judiciaires, policières, etc.) souvent défaillantes. Sur le rôle de l'arbitre dans la lucha libre, lire : LEVI, Heather, The World of Lucha Libre. Secrets, Revelations, and Mexican National Identity, Durham, Duke University Press, 2008, p. 89-95.

${ }^{9}$ Lire à ce sujet: HIER, Sean P., Moral Panic and the Politics of Anxiety, Abingdon, Ed. Routledge, 2011. Les sources du concept se trouvent dans : COHEN, Stanley, Folk Devils and Moral Panics: The Creation of the Mods and Rockers, Abingdon, Ed. Routledge, 2011 (1972).
} 
pauvre père. Le quatrième film, El Enmascarado de Plata, est celui qui ouvre le plus la voie à la naissance du nouveau genre filmique, puisqu'il fait de son héros principal (un authentique lutteur dans la vie réelle, El Médico Asesino) un fléau du crime et de l'injustice, qui utilise sa force pour défendre les faibles et les innocents. À l'instar de ce qui se passe sur les rings, le cinéma de catch propose dès lors de décrire, film après film, l'affrontement limpide du Bien et du Mal.

L'année 1954 voit l'arrivée du premier héros récurrent, La Sombra Vengadora, un personnage créé de toutes pièces par le prolifique scénariste Ramón Obón ; il apparaît dans quatre productions distribuées à très peu de temps d'intervalle, à la façon d'un serial américain : La Sombra Vengadora [Rafael Baledón, 1954], La Sombra Vengadora contra la Mano Negra [Rafael Baledón, 1954], El tesoro de Pancho Villa [Rafael Baledón, 1954] et El secreto de Pancho Villa [Rafael Baledón, 1954]. Deux ans plus tard, un nouveau film, Ladrón de cadáveres [Fernando Méndez, 1956] apporte une pierre supplémentaire à l'édifice en construction, puisque l'on y retrouve un lutteur masqué combattant le crime, mais, cette fois-ci, l'ennemi est un scientifique fou qui enlève des catcheurs et leur remplace le cerveau par celui d'animaux pour les rendre invulnérables (!). Désormais, l'univers du film d'action dans lequel avait commencé à s'insérer le cinéma de lucha libre se combinait à celui des films de monstres et de science-fiction fantaisiste, qui se mettait à connaître un fort engouement ${ }^{10}$. Cette ratatouille générique fut ensuite abondamment servie au public populaire mexicain, les producteurs veillant à changer de temps en temps les dosages et les ingrédients pour déjouer la lassitude des spectateurs.

\section{Et Dieu créa Santo}

Tandis que le cinéma de lucha libre prenait forme, le monde du catch mexicain voyait s'affirmer la popularité de Santo, devenu en quelques années une véritable idole des rings. Il était donc presque inévitable que les deux trajectoires finissent par se croiser et que Santo soit récupéré par l'industrie du cinéma.

Santo, de son vrai nom Rodolfo Guzmán Huerta, débute sa carrière de catcheur en 1935 mais n'adopte pas immédiatement son nom de scène. Combattant d'abord à visage découvert en tant que Rudy Guzmán, il devient lutteur masqué en 1936 et se fait appeler El Hombre Rojo. Comme sa carrière ne décolle pas, il s'invente une nouvelle identité et devient El Murciélago II, mais le Murciélago premier du nom, une des vedettes de l'époque, l'oblige à renoncer à ce pseudonyme. En 1942, il change donc une dernière fois de nom de scène et devient Santo. C'est avec ce personnage, qui est initialement un rudo, qu'il se met enfin à goûter au succès. Même s'il lutte fréquemment en binôme au cours des années 1940 et 1950 (la pratique était alors à la mode), sa popularité finit toujours par éclipser celle de ses partenaires et sa renommée dépasse les frontières du Distrito Federal pour devenir nationale. Bientôt, cette célébrité éveille l'intérêt d'entrepreneurs culturels qui cherchent à exploiter les droits dérivés de l'image du lutteur.

${ }^{10}$ COTTER, Robert Michael, The Mexican Masked Wrestler and Monster Filmography, Jefferson, McFarland \& Company, 2005, p. 27. 
Au début des années 1950, Santo se transforme ainsi une première fois en personnage de fiction, non pas au cinéma, mais dans la presse. En 1952, un prolifique auteur de bandes dessinées, romans-photos, scénarios et radionovelas, José G. Cruz, lui fait signer un contrat grâce auquel le lutteur masqué devient le héros d'une publication périodique, Santo, El Enmascarado de Plata (elle se présente sous la forme d'un roman-photo d'une trentaine de pages imprimées en sépia). Celle-ci commence par être hebdomadaire mais la demande est telle que José G. Cruz se met à en sortir trois numéros par semaine. Comme il s'agit avant tout d'une publication destinée à la jeunesse, Santo y incarne non pas un rudo, mais un técnico, et ses aventures imaginaires ne se déroulent pas dans l'espace clos d'un ring, mais en plein air, dans un univers fantaisiste, pour ne pas dire surréaliste, tant les histoires inventées par José G. Cruz semblent relever de l'écriture automatique. Santo, qui ne quitte jamais sa tenue de lutteur masqué, y combat inlassablement le crime et le Mal, dont les représentants, épisode après épisode, prennent d'innombrables visages. Preuve de son succès, la revue est éditée jusque dans les années 1980 (le tirage maximal dépassera les 500000 exemplaires par numéro ${ }^{11}$ ) et elle réussit à s'exporter dans plusieurs pays d'Amérique latine ${ }^{12}$.
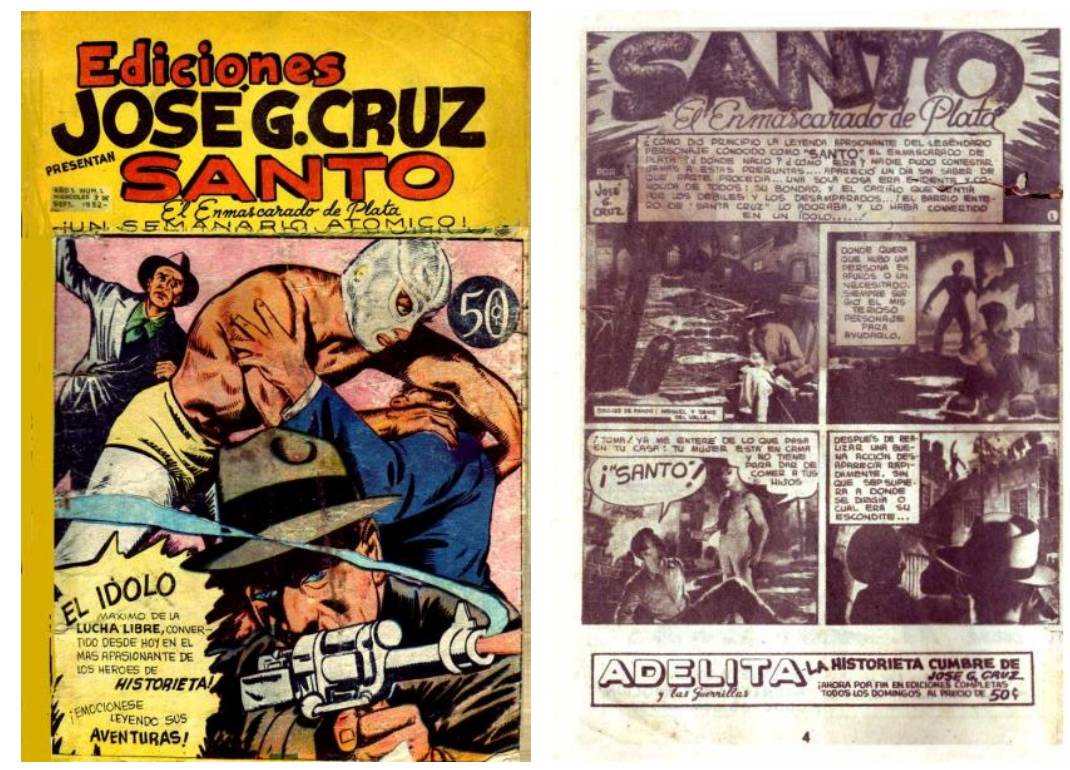

Couverture et première page du premier numéro de Santo, El Enmascarado de Plata (3 septembre 1952, Ed. José Cruz, Mexico).

L’industrie cinématographique, pour sa part, s'intéresse elle aussi de façon précoce à Santo, et le catcheur aurait dû figurer à l'affiche de l'un des tout premiers films de lucha libre, El Enmascarado de Plata [1954], dont le scénariste était d'ailleurs José G. Cruz. Au dernier moment, cependant, Santo décida de se retirer du projet car il jugeait la production peu sérieuse. Pendant plusieurs années, le cinéma de lucha libre se développa sans faire appel à Santo, et l'industrie essaya même de créer ses propres héros, en inventant, comme nous l'avons vu, les personnages de Huracán Ramírez et de La Sombra Vengadora. Mais, en 1958, Santo finit par accepter la proposition de tourner dans deux longs métrages : Santo

${ }^{11}$ FERNÁNDEZ, Alvaro A., op. cit., p. 105.

12 À la fin des années 1970, la maison d'édition colombienne ICAVI a ainsi réédité l'intégralité de la série. 
contra cerebro del mal [Joselito Rodríguez, 1961] et Santo contra hombres infernales [Joselito Rodríguez, 1961].

\section{Santo à La Havane}

Les initiateurs du projet sont le producteur mexicain Enrique Zambrano et le scénariste, acteur et catcheur d'origine espagnole Fernando Oses. Ils entendent exploiter un filon sur lequel d'autres avant eux se sont déjà lancés, et sont convaincus du potentiel commercial du genre. Au cours de la seule année 1957, huit films de catch sortent sur les écrans mexicains, signe de l'engouement croissant du public pour ce type de divertissement. Il faut néanmoins préciser que toutes ces oeuvres sont de petites productions, tournées en deux ou trois semaines avec des budgets dérisoires, et qu'elles sont distribuées dans les salles les moins prestigieuses, situées dans les quartiers les plus populaires de la capitale ainsi que dans les villes de province (pour Nelson Carro, le public des films de catch est d'ailleurs essentiellement provincial, et même rural $^{13}$ ). Il existe, dans les années 1950, une division stricte entre les cinémas de première, seconde ou troisième exclusivité, et les films de lucha libre ne peuvent prétendre accéder aux grandes salles de la capitale, dont les écrans sont occupés soit par les films hollywoodiens, soit par les productions mexicaines affichant les plus grandes stars du cinéma mexicain de l'époque, comme María Félix ou Jorge Negrete. Les deux projets de longs métrages qui sont proposés à Santo s'inscrivent dans le cadre d'un cinéma pauvre, sous-financé, techniquement déficient, artistiquement bâclé, dont les promoteurs ont pour seule ambition de faire un bon coup. Il s'agit pour eux d'investir une petite quantité d'argent (ils n'ont pas accès à des financements importants) pour récolter rapidement quelques bénéfices et enchaîner sur un nouveau projet tout aussi modeste. Il faut dire que le contexte n'était plus très favorable à la production mexicaine.

À la fin des années 1950, le cinéma local a déjà vécu ses meilleures années et la crise qui frappe le secteur oblige de nombreuses sociétés à mettre la clé sous la porte. L'une des raisons de cette crise, outre l'épuisement des formules des années 1930 et 1940 et les blocages syndicaux, est la concentration de la distribution entre les mains de l'entrepreneur américain William O. Jenkins, qui avait pour politique de favoriser les films hollywoodiens au détriment des productions mexicaines ${ }^{14}$. Seules subsistent, pour l'essentiel, de petites structures qui se sont spécialisées par défaut dans la série $B$ ou $Z$ (un enfer cinématographique également baptisé «mexploitation cinema», par référence au cinéma d'exploitation américain ${ }^{15}$ ). Les deux premiers films de Santo appartiennent clairement à

${ }^{13}$ CARRO, Nelson, El cine de luchadores, Mexico, Ed. Filmoteca UNAM, 1984, p. 47.

${ }^{14}$ Cf. VEGA ALFARO, Eduardo de la, "Origines, développement et crise du cinéma parlant (1929-1964) », in PARANAgUA, Paulo Antonio (coord.), Le cinéma mexicain, Paris, Ed. Centre Pompidou, 1992, p. 111. Pour une analyse plus approfondie, lire : CONTRERAS TORRES, Miguel, Mexico, El libro negro del cine mexicano, Mexico, Ed. Hispano-Continental Films, 1960.

15 Sur cette production, lire: GREENE, Doyle, Mexploitation cinema. A Critical History of Mexican Vampire, Wrestler, Ape-Man and Similar Films, 1957-1977, Jefferson, McFarland \& Company, 2005. Certains chercheurs utilisent également le terme de "Latsploitation», qui englobe pour sa part l'ensemble de la production latinoaméricaine. Sur les différences terminologiques entre «exploitation», "mexploitation» et «Latsploitation», lire: RUÉTALO, Victoria, TIERNEY, Dolores, "Reinventing the Frame : Exploitation and Latin America », in RUÉTALO, Victoria, TIERNEY, Dolores, Latsploitation, Exploitation Cinemas, and Latin America, New York, Ed. Routledge, 2009, p.1-12. L'ouvrage de référence sur le cinéma d'exploitation américain est: 
cette deuxième catégorie et sont emblématiques de la spirale descendante dans laquelle est alors engagé le cinéma mexicain. Afin de réduire les coûts de production, le producteur principal, Enrique Zambrano, décida, comme cela était fréquent à l'époque, de délocaliser le tournage à Cuba et d'y trouver un coproducteur. Les artistes et techniciens cubains, qui évoluaient dans un environnement professionnel beaucoup plus précaire que celui de leurs homologues mexicains, étaient en effet beaucoup moins bien payés qu'eux. L'autre solution pour réduire les coûts consista à tourner très vite, au détriment de la qualité technique, et à réaliser deux films lors du même séjour. Les noms figurant au générique de Santo contra cerebro del mal et Santo contra hombres infernales sont quasiment identiques, tant pour les acteurs que pour les techniciens, et le visionnage des deux films confirme que le tournage des deux long métrages eut lieu en même temps, à La Havane (vraisemblablement quelques semaines avant la Révolution, en novembre ou décembre 1958). Plusieurs plans insistants sur un hôtel de la ville, le Colina, situé dans le Vedado, laissent penser qu'il s'agit en réalité d'un peu de publicité faite au propriétaire, sans doute en échange d'une ristourne sur le prix des chambres, ce qui donne une idée des limitations économiques auxquelles se heurtait la production.
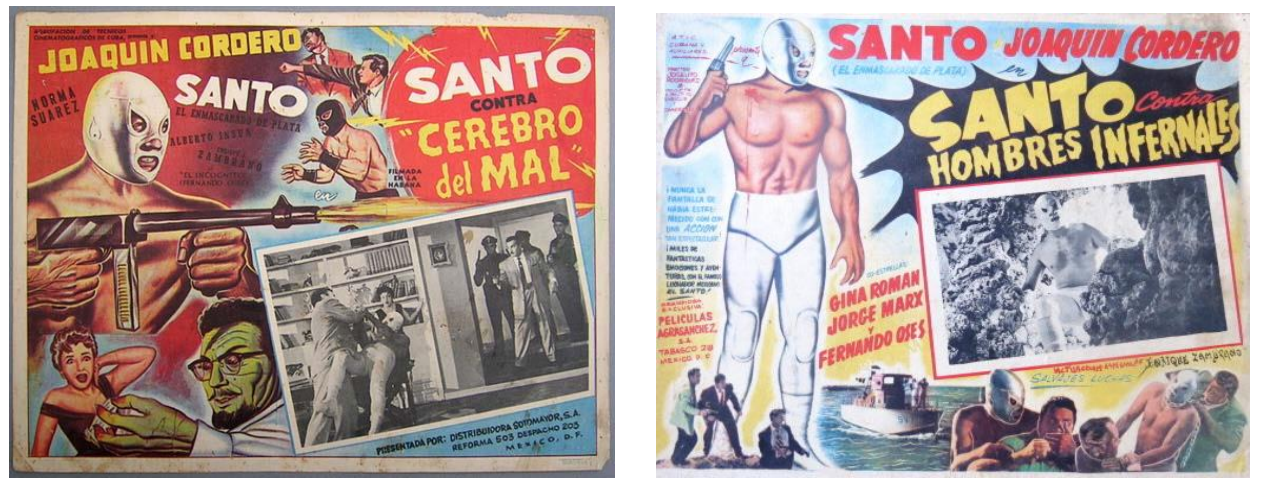

Même si les deux longs métrages mettent en vedette Santo dans leurs affiches et leurs titres respectifs, le lutteur n'apparaît finalement qu'assez peu à l'écran et n'incarne qu'un personnage secondaire, qui ne s'appelle d'ailleurs pas Santo, mais El Enmascarado. C'est l'une des premières incohérences de ces deux productions qui n'entretiennent que de lointains rapports avec l'esprit logique. Dans Santo contra cerebro del mal, le catcheur est introduit dès la première séquence, et le scénario ne se donne même pas la peine de le présenter car il est présupposé que le spectateur connait déjà le héros, pour l'avoir vu combattre sur le ring ou bien pour avoir suivi ses aventures graphiques. Le film n'est ici qu'un produit dérivé de l'univers fictionnel élaboré par José G. Cruz dans ses romansphotos (ou fotocomics), lui-même dérivé de l'univers théâtral du ring. Le personnage est si connu que les auteurs du film peuvent se permettre, pour sa toute première apparition à l'écran, de renverser son rôle habituel, en faisant de lui un méchant au service d'un scientifique fou. Dans la première séquence, Santo est poursuivi par une bande de malfrats qui parviennent à l'assommer et à l'emmener dans le laboratoire du diabolique professeur Campos. Ce dernier lui injecte un produit spécial puis l'expose à des rayons tout aussi spéciaux, qui le transforment en un être docile et manipulable à souhait. Désormais, il est contraint de mettre sa force herculéenne au service du dangereux scientifique. À la moitié du film, un autre lutteur masqué, El Incógnito, parvient néanmoins à tirer Santo de son état

SCHAEFER, Eric, Bold! Daring! Shocking! True: A History of Exploitation Films, 1919-1959, Durham, Duke University Press, 1999. 
de soumission et tous deux unissent leurs forces à celles de la police cubaine pour mettre en déroute le professeur Campos alors que ce dernier cherche à vendre la formule d'inventions destructrices à une puissance étrangère.

Le double emploi de Santo, qui agit d'abord en tant que méchant avant de rejoindre les forces du bien, était en fait à l'image de la double identité qu'il s'était forgée sur le ring et dans les magazines à sa gloire. Dans le premier univers, il continuait à être un rudo alors que dans ses romans-photos, il apparaissait comme un técnico. Santo contra cerebro del mal exprime la dualité du personnage à l'époque et annonce également son basculement du côté du bien et sa conversion définitive en técnico, qui se produit sur les rings au tout début des années 1960. En ce sens, le film constitue un point d'inflexion dans la carrière du catcheur. Dans le deuxième long métrage, Santo contra hombres infernales, qui sort quelques semaines après le premier, la mutation est opérée, et Santo est, dès le début, un adjuvant de la police, qui combat cette fois-ci des contrebandiers. L'histoire est encore plus décousue et encore plus mal mise en scène que dans le premier opus, mais elle a le mérite de davantage mettre en avant Santo, qui n'est plus affublé de El Incógnito. Il n'en reste pas moins surprenant de constater la timidité avec laquelle les auteurs du film (celui-ci est réalisé par Joselito Rodríguez, qui a déjà tourné le premier Huracán Ramírez en 1953) utilisent leur vedette principale, censée attirer les foules dans les salles obscures. Santo n'exploite pas non plus vraiment ses talents de catcheur (les bagarres auxquelles il participe sont mollement orchestrées) et jamais il n'apparaît sur un ring. Autre absence à déplorer: celle des monstres, qui deviendront par la suite indissociables de l'univers filmique de Santo. On trouve bien dans Santo contra cerebro del mal un personnage de scientifique maléfique, mais son laboratoire secret ne produit aucune créature inquiétante. Quant au deuxième long métrage, il met en scène des contrebandiers tout à fait communs.

Pour toutes ces raisons, Santo contra cerebro del mal et Santo contra hombres infernales diffèrent nettement du reste de la filmographie du lutteur masqué et apparaissent comme un premier galop d'essai mal maitrisé, une répétition hésitante avant que la carrière cinématographique de Santo ne démarre vraiment, grâce à son troisième film, Santo contra los zombies [Benito Alazraki, 1962]. Dans ce long métrage, tous les ingrédients qui ont fait le succès des aventures graphiques de Santo se trouvent réunis : scènes de catch à gogo et monstres en pagaille, dans une lutte à mort entre le Bien et le Mal.

\section{Mexploitation expérimentale}

Malgré toutes les lacunes que je viens d'évoquer, les deux premiers films avec Santo furent des succès publics et permirent à de nouveaux projets de voir le jour. S'il est impossible de déterminer exactement les raisons d'un tel succès, il est néanmoins possible d'avancer quelques hypothèses.

On signalera pour commencer que les deux films de Joselito Rodríguez ne se contentaient pas de reprendre un personnage et un univers fictionnel déjà connus, gages de reconnaissance et d'acceptation par le public, mais s'inspiraient également d'un genre cinématographique en vogue quelques années auparavant, à savoir le cinéma de rumberas. On retrouve en effet dans les deux titres des numéros dansés filmés dans des cabarets 
célèbres de La Havane, comme le Capri, ainsi qu'un passage musical où un trio chante une chanson cubaine. Tous ces éléments étaient déjà présents dans les films de cabareteras, qui étaient parfois tournés en coproduction à La Havane et dont les actrices étaient souvent caribéennes ${ }^{16}$. D'une certaine façon, Santo contra cerebro del mal et Santo contra hombres infernales réactivent chez le spectateur mexicain de l'époque le souvenir d'un genre qui avait alors disparu depuis peu des écrans, victime notamment de la censure morale, et lui proposent de retrouver un univers familier et séducteur, associé à l'exotisme tropical. Comme les films de rumberas, les deux premiers films de Santo mettent d'ailleurs le corps de l'acteur en avant et l'offrent aux regards des spectateurs. Il ne s'agit plus du corps féminin des danseuses de cabarets, corps désirés et désirants, mais de corps masculins, souffrants et agissants. On assiste donc dans ces films, à partir de repères familiers, à un glissement vers un nouvel univers cinématographique, comme s'il s'agissait d'opérer une transition en douceur, de dire adieu aux films de rumberas tout en préparant l'avènement du cinéma de lucha libre.

Le plaisir que procure la vision des deux films tient également à leur caractère totalement fantaisiste et irréaliste, qui est, depuis Méliès, la marque du cinéma de divertissement et d'évasion. Jusqu'alors, le cinéma mexicain n'avait jamais brillé par son souci du réel, que ce soit dans ses œuvres les plus ambitieuses (Emilio Fernández) ou dans ses productions les plus commerciales (Juan Orol), mais Santo contra cerebro del mal et Santo contra hombres infernales creusent volontairement le sillon ouvert l'année précédente par des films tels que Ladrón de cadáveres [Fernando Méndez, 1957], El vampiro [Fernando Méndez, 1957] ou La maldición de la momia azteca [Rafael Portillo, 1957]. L'histoire de Santo contra cerebro del mal, en plus d'être totalement improbable, est racontée en dépit du bon sens et trahit un désintérêt profond pour les notions de crédibilité et de plausibilité. Il est évidemment possible d'invoquer l'incompétence technique et artistique des auteurs (un facteur à ne jamais négliger) ainsi que leur mépris fondamental du public, supposé être incapable de séparer le bon grain de l'ivraie cinématographique, mais on peut aussi y voir une volonté plus ou moins consciente de s'affranchir des contraintes étouffantes de l'esprit logique pour vagabonder sur les terres de l'imaginaire et de l'onirisme. Nous avons déjà souligné ce que les romans-photos de José G. Cruz, publiés à un rythme étourdissant, devaient à l'écriture automatique et, d'une certaine façon, les deux films de Joselito Rodríguez, dans leur accumulation d'incohérences scénaristiques et d'aberrations de mise en scène, finissent par attraper le spectateur dans un récit qui se déroule comme dans les rêves, où les événements se produisent en dehors de tout principe de causalité apparent. Une logique finit par s’imposer qui n'est pas celle de la logique, et qui nous amène à suivre avec délectation une histoire totalement abracadabrantesque. L'indigence même de la production contribue à produire cet effet, notamment lorsque, par souci d'économie, le réalisateur choisit de répéter certains plans et certaines séquences, d'abord au sein d'un même film, puis d'un film à l'autre. Cette pratique, qui s'inspire des techniques de réalisation des romans-photos de la série Santo, El Enmascarado de Plata, dont les auteurs procèdent essentiellement par collage et juxtaposition d'éléments visuels prêts à l'emploi, crée un effet hypnotique sur le spectateur, qui a le sentiment que le film bégaie et tourne en boucle. Il serait fastidieux de repérer un à un tous ces moments de redite et d'autocannibalisme, mais ils sont étonnamment nombreux, que ce soit dans Santo contra cerebro del mal ou dans Santo contra hombres infernales.

${ }^{16}$ Lire à ce sujet : AMIOT, Julie, Le mélodrame mexicain dans ses rapports avec Cuba (1938-1958): enjeux esthétiques et critiques, thèse soutenue en 2003 à l'université Lumière Lyon 2 sous la direction de M. Jean-Claude Seguin. Texte accessible en ligne: http://theses.univ-lyon2.fr/documents/lyon2/2003/amiot_j (dernière consultation : 03/10/2013). 
L'effet le plus surprenant se produit lors du visionnage successif des deux films, qui laisse apparaître un important recyclage des images du premier dans le second (à moins qu'il ne s'agisse de l'inverse, les conditions exactes du montage nous étant inconnues). Certains réemplois se limitent à un plan fugace, mais, dans d'autres cas, ce sont des séquences entières qui sont reprises, dotées d'un sens différent dans le nouveau récit. Un exemple frappant concerne la séquence où un personnage sort de l'hôtel Colina. Le même acteur sort du même hôtel et monte dans la même voiture dans Santo contra cerebro del mal et dans Santo contra hombres infernales. Dans le premier film, la séquence commence à la $40^{\mathrm{e}}$ minute, tandis que dans le second, elle démarre à la $13^{\mathrm{e}}$ minute.

Au cours de la séquence suivante, l'homme que l'on vient de voir sortir de l'hôtel (un gangster) se fait agresser dans un terrain vague par les membres d'une bande rivale. Ce n'est pas le même personnage dans les deux films, il ne se fait pas attaquer pour les mêmes raisons, mais rien de tout cela ne semble gêner le réalisateur, qui renouvelle même l'opération à plusieurs reprises. En effet, dès qu'une situation dramaturgique se répète d'un film à l'autre (une poursuite en voitures, un règlement de comptes, une filature, etc.), Joselito Rodríguez n'hésite pas à piocher dans le stock d'images du premier film, comme s'il s'agissait d'une banque d'organes.

À certains moments, on retrouve des séquences proches mais pas totalement identiques. Il semble, en réalité, que les producteurs aient récupéré dans Hombres infernales des prises inutilisées de Cerebro del mal. C'est le cas, en particulier, dans deux séquences musicales, de simples pauses dans le récit, à la fonction uniquement ornementale. La première (23' et 51') montre des danseurs de flamenco dont la présence est assez incongrue (les rumberas n'ont plus à l'époque les faveurs du public, et le réalisateur ne sait pas vraiment par quoi les remplacer), tandis que la deuxième (43' et 22') introduit un trio cubain, qui interprète des boléros à la terrasse d'un café. Dans le premier cas, les danseurs sont les mêmes dans les deux films mais interprètent un numéro différent (on remarque qu'ils ont changé de costume). Quant à la deuxième séquence musicale, elle donne à entendre la même chanson, interprétée au même endroit par le même trio, mais le type de plan a changé dans la version de Hombres infernales (on passe d'un plan moyen à un plan général).

Dans cette dynamique de recyclage totalement décomplexée, il arrive également que soient repris des dialogues entiers, les auteurs se contentant de légères retouches pour adapter le texte à la nouvelle intrigue. À la fin de Cerebro del mal, lorsque la police a réussi, avec l'aide de Santo et de El Incógnito, à faire échouer les plans machiavéliques du savant fou, le commissaire qui a mené l'enquête et son lieutenant accompagnent à l'aéroport deux des personnages, ainsi que les lutteurs masqués. Tandis que les passagers embarquent et que l'appareil décolle vers une destination inconnue, le commissaire et le lieutenant échangent quelques propos :

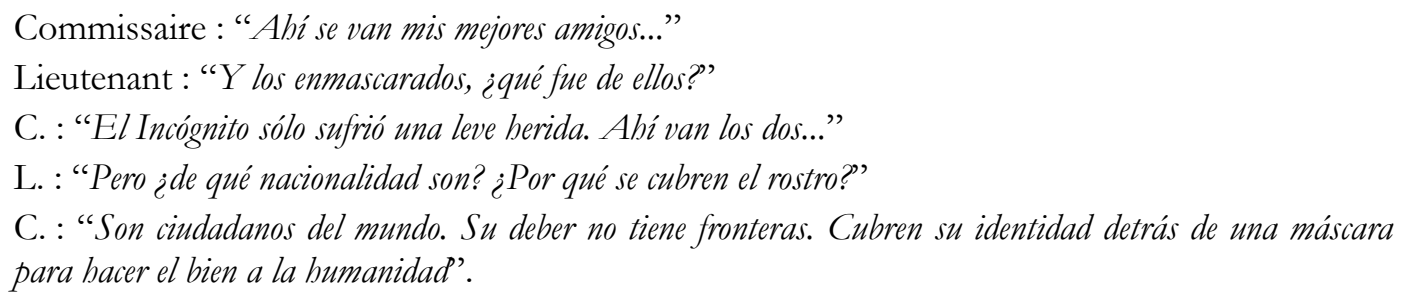


Dans Hombres infernales, El Incógnito (interprété par Fernando Osés, par ailleurs auteur du scénario) a disparu du générique et la police ne peut plus compter que sur Santo pour tenter de démanteler un réseau de trafiquants. La séquence finale propose des images semblables à celles de Cerebro del mal, mais montées de manière très légèrement différente ; le commissaire et son lieutenant sont à nouveau à l'aéroport et le spectateur imagine qu'ils ont accompagné Santo jusqu'à son avion (le catcheur reste invisible). Tandis que les passagers embarquent et que l'appareil décolle vers une destination inconnue, le commissaire et le lieutenant échangent quelques propos :

Commissaire : "Ahi se van mis mejores amigos... Te felicito, biciste un buen trabajo, tienes muy buena puntería".

Lieutenant: "Tuve que herirlo para que no sospechara nada de mí. Y El Enmascarado, ¿qué fue de él?".

C. : "El agente sólo sufrió una leve herida. Ahi van los dos...".

L. : "Pero ¿de qué nacionalidad es? ¿Por qué se cubre el rostro?".

C. : "Es ciudadano del mundo. Su deber no tiene fronteras. Cubre su identidad detrás de una máscara para hacer el bien a la bumanidad".

Bien entendu, les deux films se concluent par la même image, celle d'un avion quittant Cuba. D'une certaine façon, cette image est prémonitoire puisque Santo et les autres lutteurs ne remettront plus jamais les pieds sur l'île.

\section{Conclusion}

Les deux premiers films de la série des Santo sont de toute évidence des films très mineurs, pour ne pas dire d'authentiques churros, et nul ne songerait à leur attribuer une valeur artistique que leurs auteurs n'ont d'ailleurs jamais prétendu leur donner. Il n'en reste pas moins que ces deux opus présentent un intérêt historique certain, puisqu'ils posent les fondements de l'une des sagas cinématographiques les plus durables de l'histoire du cinéma, en testant la recette de son succès et en faisant définitivement basculer Santo du côté de la veuve et de l'orphelin; ils constituent également le point d'inflexion entre deux époques du cinéma populaire mexicain et marquent le passage de flambeau entre les films de rumberas, dominants dans les années 1940 et 1950, et ceux, encore plus ignorés par les élites culturelles, de luchadores, un genre qui attirera les masses plébéiennes jusque dans les années 1980. Au-delà de ces aspects, on observe surtout dans ces deux films une surprenante et quasi expérimentale mise à nu de leur propre matière textuelle, une mise en évidence du langage cinématographique lui-même, dont on sait qu'il repose en grande partie sur la mécanique du montage et de l'assemblage de plans. Avec Santo, toutes les coutures que le cinéma classique s'évertue habituellement à dissimuler sont soulignées, dévoilées, exhibées au regard du spectateur qui, comme au catch, n'est plus dupe de rien. «Le cinéma, ce n'est rien d'autre que du meccano, une simple opération de bricolage dont voici les boulons » semblent vouloir nous dire le réalisateur et son héros masqué. Soit, exprimé avec les mots du catch : «Le cinéma, c’est du chiqué ». 